\title{
Camada de resíduos florestais e pressão de preconsolidação de dois latossolos
}

\author{
Arystides Resende Silva ${ }^{(1)}$, Moacir de Souza Dias Junior ${ }^{(1)}$ e Fernando Palha Leite(2)
}

(1)Universidade Federal de Lavras, Dep. de Ciência do Solo, Caixa Postal 3037, CEP 37200-000 Lavras, MG. E-mail: arystidesrs@yahoo.com.br, msouzadj@ufla.br (2)Celulose Nipo-Brasileira S.A., CEP 35195-000 Belo Oriente, MG. E-mail: fernando.leite@cenibra.com.br

\begin{abstract}
Resumo - O objetivo deste trabalho foi avaliar o efeito da camada de resíduos da colheita sobre a pressão de preconsolidação, com o uso dos modelos de capacidade de suporte de carga de um Latossolo Amarelo e de um Latossolo Vermelho. Foram coletadas 20 amostras indeformadas, em cada tipo de solo, à profundidade de 10-13 cm, na linha de tráfego do "forwarder", depois de este ter trafegado duas e oito vezes em três condições de disposição de resíduo: sobre a galhada $(\mathrm{G})$; sobre a galhada mais a casca (GC); sobre o solo sem resíduo (SR); e onde não houve tráfego do "forwarder" (ST). As amostras indeformadas foram utilizadas nos ensaios de compressão uniaxial. Determinaram-se, também, textura, matéria orgânica e densidade de partículas dos solos. O tráfego sobre os resíduos florestais atenuou a compactação nos solos estudados, principalmente na condição de galhada + casca (GC), onde a pior situação foi a condição de solo limpo (SR) nos dois tipos de solos. Quando a intensidade de tráfego foi aumentada de duas para oito vezes, houve maior compactação do solo.
\end{abstract}

Termos para indexação: Eucalyptus, compactação do solo, tráfego, mecanização florestal.

\section{Forest residuals layer in the preconsolidation pressure of two Oxisols}

\begin{abstract}
The objective of this work was to evaluate the effect of forest residue on the preconsolidation pressure using the load support capacity models of a Typic Haplustox and aTypic Acrustox. Twenty undisturbed soil samples were collected in each soil type at 10-13 cm depth, in the traffic line of the forwarder after it trafficked over residue disposition, two and eight times, in three conditions, being the traffic performed: on brushwood (G); on the brushwood and bark (GC); on soil without residue (SR); and on soil with no traffic (ST). Undisturbed soil samples were used in the uniaxial compression tests. The particle size distribution, organic matter and particle density were also determined. The traffic over forest residues attenuated the soil compaction in the studied soils, mainly in the condition of brushwood and bark (GC); soil without residue (SR) was the worse condition in the two soil types. As traffic intensity increased from two to eight times, a larger soil compaction ocurred.
\end{abstract}

Index terms: Eucalyptus, soil compaction, traffic, forest mechanization.

\section{Introdução}

A compactação de solos florestais tem ocorrido em virtude de operações florestais mecanizadas, que envolvem o tráfego intensivo de máquinas sobre o solo (Dias Junior \& Pierce, 1996; Czyz, 2004; Dias Júnior et al., 2005; Hamza \& Handerson, 2005).

Operações de colheita e baldeio da madeira (Dias Junior et al., 1999, 2003), principalmente quando o solo se encontra em condições inadequadas de umidade (Arvidsson et al., 2003; Chamen et al., 2003; Dias Junior et al., 2005; Raper, 2005), causam compactação e podem prejudicar a produção desses solos (Canillas \& Salokhe, 2002; Czyz, 2004).
O uso sustentável do solo em áreas florestais está relacionado ao tráfego de veículos durante as operações, principalmente de colheita e extração da madeira (Dias Junior et al., 2002, 2003, 2005), que visam, em áreas de produção de celulose, à abastecer fábricas de madeira durante o ano.

Uma alternativa para minimizar o impacto do tráfego na estrutura dos solos sob exploração florestal é o uso de resíduos florestais (Seixas et al., 1998). A finalidade do uso desses resíduos é que eles distribuam as pressões aplicadas, de tal forma a evitar que a capacidade de suporte de carga do solo seja superada, o que reduziria o risco da compactação e a formação de sulcos pelos rodados das máquinas (McMahon \& Evanson, 1994). 
O objetivo deste trabalho foi avaliar o efeito da camada de resíduos florestais sobre a pressão de preconsolidação do solo, com uso de modelos de capacidade de suporte de carga, em dois tipos de Latossolo.

\section{Material e Métodos}

Este trabalho foi realizado em áreas experimentais da Celulose Nipo-Brasileira S.A. (Cenibra), na região do Rio Doce, no projeto Marola, no Município de Belo Oriente, MG, a $19^{\circ} 18^{\prime} 23^{\prime \prime} \mathrm{S}$ e $42^{\circ} 25^{\prime} 31^{\prime \prime} \mathrm{W}$ com altitude de $300 \mathrm{~m}$; e na de Guanhães, no projeto Jambreiro, no município de São João Evangelista, MG, a 18³4'12"S e 4252'53"W, com altitude de $860 \mathrm{~m}$.

O solo da região do Rio Doce foi classificado como Latossolo Amarelo (LA), textura argilosa e o da região de Guanhães, como Latossolo Vermelho (LV), textura argilosa.

Foram demarcadas parcelas de $36 \mathrm{~m}$ de comprimento e $4 \mathrm{~m}$ de largura, que correspondem à entrelinha de 12 árvores de eucaliptos. A colheita florestal foi realizada manualmente, com o uso de motosserra, e a extração da madeira foi feita com "forwarder" (marca Valmet, modelo 636 S), com tara de $11,9 \mathrm{t}$, carregado com $9 \mathrm{~m}^{3}$ de madeira (densidade de $480 \mathrm{~kg} \mathrm{~m}^{-3}$ ), que trafegou nas seguintes condições de disposição dos resíduos: tráfego sobre a galhada (G); tráfego sobre a galhada mais a casca (GC); tráfego sobre o solo sem resíduo (SR); e sem tráfego do "forwarder" (ST). Em cada condição de disposição de resíduo, foram coletadas 20 amostras na linha de tráfego do "forwarder", à profundidade de 10-13 cm, após este ter trafegado duas e oito vezes na mesma entrelinha, que totalizaram 320 amostras (20 amostras x 4 condições de resíduos x 2 condições de passadas x 2 regiões). As amostras foram parafinadas no campo, para evitar alterações na umidade, do momento da coleta até o da realização do ensaio de compressão uniaxial no laboratório.

Os ensaios de compressão uniaxial foram realizados nas amostras com um consolidômetro da marca Boart Longyear, com aplicação de pressão por meio de ar comprimido na seguinte ordem: 25, 50, 100, 200, $400,800,1.600 \mathrm{kPa}$. Cada pressão foi aplicada até que $90 \%$ da deformação máxima fosse atingida (Taylor, 1948) e, após essa condição ter sido atingida, aplicou-se uma nova pressão. Para cada umidade de operação do "forwarder", as pressões de preconsolidação foram obtidas de acordo com Dias Junior \& Pierce (1995) e plotadas nos modelos de capacidade de suporte de carga de cada região, obtidos por Dias Junior et al. (2005), para determinar o efeito dos resíduos sobre a pressão de preconsolidação.

As amostras deformadas foram obtidas pela coleta de solo, das porções superiores e inferiores dos anéis de amostragem, nas quais foram determinados os teores de matéria orgânica (Raij \& Quaggio, 1983), textura (Day, 1986) e densidade de partículas (Blake \& Hartge, 1986) (Tabela 1).

Os resultados das análises das características físicas do solo foram submetidos à análise de variância, e a comparação das médias das profundidades foram feitas pelo teste de Scott-Knott $(\mathrm{p}<0,05)$.

\section{Resultados e Discussão}

Para avaliar os efeitos das diferentes condições de disposição de resíduos sobre a pressão de preconsolidação, utilizaram-se modelos de capacidade de suporte de carga obtidos por Dias Junior et al. (2005) para as regiões onde este trabalho foi realizado (Figura 1).

Considerando-se a pressão de preconsolidação como indicativo da máxima pressão aplicada ao solo no passado (Holtz \& Kovacs, 1981; Dias Junior, 1994), e um indicador da suscetibilidade do solo à compactação (Peng et al., 2004), a Figura 1 foi dividida em três regiões, para avaliar o efeito do tráfego sobre a pressão de preconsolidação, de acordo com Dias Junior et al. (2005). As regiões consideradas são: a) região onde os valores da pressão de preconsolidação determinados após o tráfego forem maiores que o limite superior do intervalo de confiança, sendo considerada a região com compactação do solo adicional; b) região onde as valores da pressão de preconsolidação determinados após o tráfego estão entre os limites de confiança inferior e

Tabela 1. Análises de caracterização física de um Latossolo Amarelo (LA), localizado na região do Rio Doce e de um Latossolo Vermelho (LV), localizado na região de Guanhães, à profundidade de $10-13 \mathrm{~cm}^{(1)}$.

\begin{tabular}{|c|c|c|c|c|c|}
\hline Solo & Argila & Areia & Silte & $\begin{array}{l}\text { Matéria } \\
\text { orgânica }\end{array}$ & $\begin{array}{c}\text { Densidade } \\
\text { de partículas } \\
\left(\mathrm{g} \mathrm{cm}^{-3}\right)\end{array}$ \\
\hline LA & $590 b$ & $343 a$ & $67 b$ & $28 \mathrm{~b}$ & $2,56 \mathrm{a}$ \\
\hline LV & $747 a$ & $183 \mathrm{~b}$ & $70 a$ & $61 \mathrm{a}$ & $2,53 \mathrm{~b}$ \\
\hline
\end{tabular}

${ }^{(1)}$ Médias seguidas de letras iguais nas colunas não diferem entre si pelo teste de Scott-Knott, a $5 \%$ de probabilidade. 
superior. Embora as amostras de solo situadas nesta região não tenham sofrido compactação, esta região indica que as amostras poderão sofrer compactação nas próximas operações de colheita, se as pressões aplicadas forem maiores que o limite superior do intervalo de confiança; c) região onde os valores da pressão de preconsolidação determinada após o tráfego são menores que o limite inferior do intervalo de confiança (Figura 2).

Tanto para a região do Rio Doce (Tabela 2) quanto para a de Guanhães (Tabela 3), a condição sem tráfego (ST) apresentou $100 \%$ das amostras com pressão de preconsolidação abaixo da estimada pelo modelo de capacidade de suporte de cargas, o que evidencia que o solo, no início do experimento, não possuía nenhuma camada compactada ou de adensamento natural.

Quando o "forwarder" trafegou duas vezes na região do Rio Doce, sobre galhada + casca (GC) e sobre galhada (G) (Tabela 2), 100\% das amostras não sofreram compactação e, na condição de solo sem resíduo (SR), 5\% das amostras foram compactadas.

Com o aumento da intensidade de tráfego do "forwarder" para oito passadas, na região do Rio Doce (Tabela 2), observou-se que o tráfego sobre galhada +

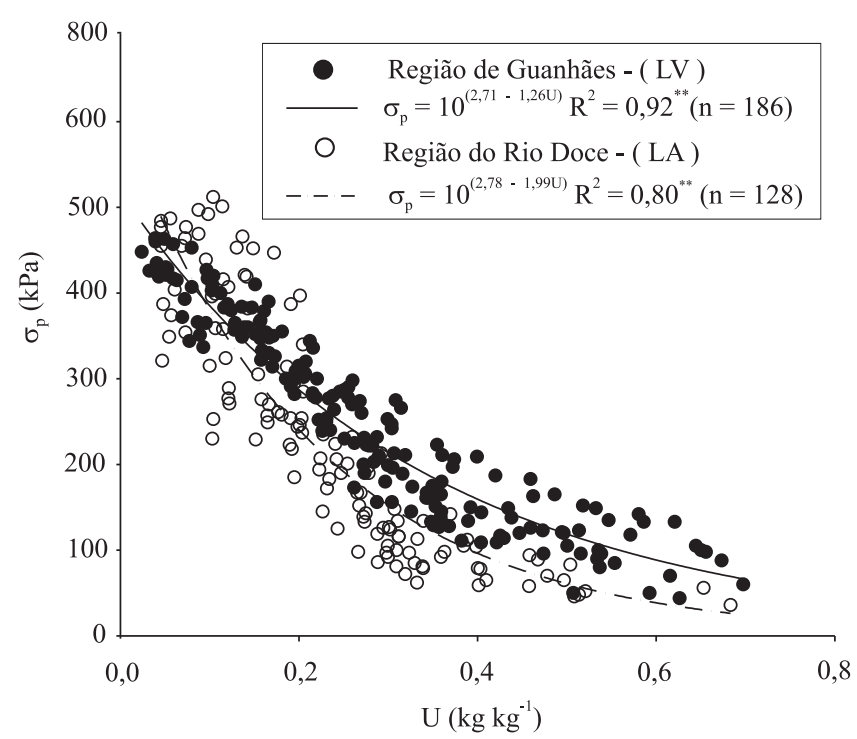

Figura 1. Modelos da capacidade de suporte de carga para o Latossolo Amarelo (região do Rio Doce) e para o Latossolo Vermelho (região de Guanhães), à profundidade de 10-13 cm. Fonte: Dias Junior et al. (2005). casca (GC) apresentou 5\% das amostras compactadas, enquanto na condição de galhada $(\mathrm{G}), 15 \%$ das amostras apresentaram compactação, e na condição solos sem resíduo (SR), $70 \%$ das amostra foram compactadas.

Observou-se que, à medida que aumentou a intensidade de tráfego do "forwarder" de duas para oito passadas, ocorreu degradação da estrutura do solo em todas as condições de disposição dos resíduos, o que é evidenciado pelo aumento dos valores das pressões de preconsolidação, principalmente para a condição de solo sem resíduo (SR) (Tabela 2). Wood et al. (1993), Seixas et al. (2003), Silva (2003), Czyz (2004) e Servadio et al. (2005) verificaram maior degradação da estrutura do solo com o aumento do número de passadas.

Apesar de a compactação ter aumentado com o aumento do número de passadas do "forwarder", na região do Rio Doce, as condições de galhada + casca (GC) e galhada $(G)$ minimizaram o efeito da compactação, sendo a condição de galhada + casca (GC) a mais eficiente (Tabela 2).

Quando o "forwarder" trafegou duas vezes na região de Guanhães, não ocorreu compactação do solo em nenhuma condição de disposição dos resíduos, entretanto, a maioria das amostras apresentou tendência à compactação (Tabela 3 ).

Com o aumento da intensidade de tráfego para oito passadas (Tabela 3), observa-se que, na condição de galhada + casca (GC), não ocorreu compactação; na condição de galhada $(\mathrm{G})$, a compactação ocorreu em $10 \%$

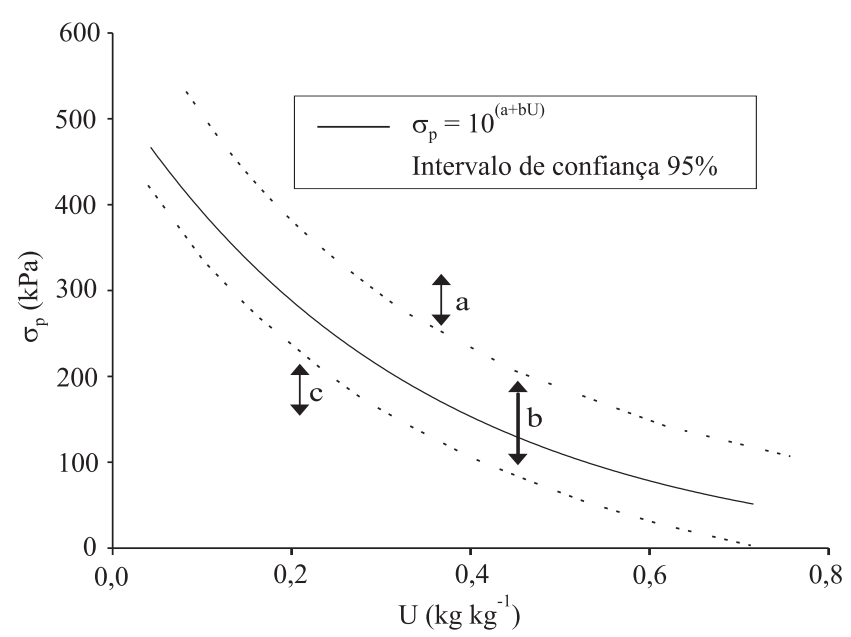

Figura 2. Critérios usados para analisar o efeito das operações mecanizadas na pressão de preconsolidação. Fonte: Dias Junior et al. (2005). 
Tabela 2. Valores médios de umidade e pressão de preconsolidação, determinados após o tráfego do "forwarder” por duas e oito vezes, em condições de disposição de resíduos, em um Latossolo Amarelo, à profundidade de 10-13 cm, na região Belo Oriente, MG, e classificação de amostras de solo, em porcentagem, de acordo com cada região "a", "b" e "c" do modelo sugerido por Dias Junior et al. (2005) $)^{(1)}$.

\begin{tabular}{|c|c|c|c|c|c|c|c|c|c|c|c|c|}
\hline \multirow[t]{2}{*}{ Região } & \multicolumn{2}{|c|}{ ST } & \multicolumn{2}{|c|}{ GC } & \multicolumn{2}{|c|}{ G } & \multicolumn{2}{|c|}{ SR } & \multirow{2}{*}{$\begin{array}{l}\text { ST } \\
(\%)\end{array}$} & \multirow{2}{*}{$\begin{array}{l}\text { GC } \\
(\%)\end{array}$} & \multirow{2}{*}{$\begin{array}{l}\mathrm{G} \\
(\%)\end{array}$} & \multirow{2}{*}{$\begin{array}{l}\text { SR } \\
(\%)\end{array}$} \\
\hline & $\begin{array}{c}\mathrm{U} \\
\left(\mathrm{kg} \mathrm{kg}^{-1}\right)\end{array}$ & $\begin{array}{c}\sigma_{\mathrm{p}} \\
(\mathrm{kPa})\end{array}$ & $\begin{array}{c}\mathrm{U} \\
\left(\mathrm{kg} \mathrm{kg}^{-1}\right)\end{array}$ & $\begin{array}{c}\sigma_{\mathrm{p}} \\
(\mathrm{kPa})\end{array}$ & $\begin{array}{c}\mathrm{U} \\
\left(\mathrm{kg} \mathrm{kg}^{-1}\right)\end{array}$ & $\begin{array}{c}\sigma_{\mathrm{p}} \\
(\mathrm{kPa})\end{array}$ & $\begin{array}{c}\mathrm{U} \\
\left(\mathrm{kg} \mathrm{kg}^{-1}\right)\end{array}$ & $\begin{array}{c}\sigma_{\mathrm{p}} \\
(\mathrm{kPa})\end{array}$ & & & & \\
\hline & \multicolumn{12}{|c|}{ Duas passadas do "forwarder" } \\
\hline a. Com compactação & & - & & & - & - & $0,27^{(2)}$ & $297^{(2)}$ & 0 & 0 & 0 & 5 \\
\hline b. Com tendência a compactar & $0,25^{(3)}$ & $125^{(3)}$ & $0,27^{(3)}$ & $205^{(3)}$ & $0,26^{(3)}$ & $210^{(3)}$ & $0,25^{(4)}$ & $262^{(4)}$ & 100 & 100 & 100 & 95 \\
\hline c. Sem compactação & - & - & - & - & - & - & - & - & 0 & 0 & 0 & 0 \\
\hline & \multicolumn{12}{|c|}{ Oito passadas do "forwarder" } \\
\hline a. Com compactação & -1 & - & $0,29^{(2)}$ & $311^{(2)}$ & $0,28^{(5)}$ & $305^{(5)}$ & $0,25^{(6)}$ & $327^{(6)}$ & 0 & 5 & 15 & 70 \\
\hline b. Com tendência a compactar & $0,25^{(3)}$ & $125^{(3)}$ & $0,27^{(4)}$ & $224^{(4)}$ & $0,26^{(7)}$ & $257^{(7)}$ & $0,24^{(8)}$ & $284^{(8)}$ & 100 & 95 & 85 & 30 \\
\hline c. Sem compactação & - & - & - & - & - & - & - & - & 0 & 0 & 0 & 0 \\
\hline
\end{tabular}

${ }^{(1)}$ ST: sem tráfego do "forwarder"; GC: presença de galhada + casca; G: presença de galhada; SR: sem resíduo. (2)Uma amostra. ${ }^{(3)}$ Média de 20 amostras. ${ }^{(4)}$ Média de 19 amostras. ${ }^{(5)}$ Média de 3 amostras. ${ }^{(6)}$ Média de 14 amostras. ${ }^{(7)}$ Média de 17 amostras. ${ }^{(8)}$ Média de 6 amostras.

Tabela 3. Valores médios de umidade e pressão de preconsolidação, determinados após o tráfego do "forwarder" por duas e oito vezes, em diferentes condições de disposição de resíduos para um Latossolo Vermelho, na profundidade de 10-13 cm, na região São João Evangelista, MG, e classificação das amostras de solo, em porcentagem, de acordo com cada região "a", "b" e "c" do modelo sugerido por Dias Junior et al. $(2005)^{(1)}$.

\begin{tabular}{|c|c|c|c|c|c|c|c|c|c|c|c|c|}
\hline \multirow[t]{2}{*}{ Região } & \multicolumn{2}{|c|}{ ST } & \multicolumn{2}{|c|}{ GC } & \multicolumn{2}{|c|}{$\mathrm{G}$} & \multicolumn{2}{|c|}{ SR } & \multirow{2}{*}{$\begin{array}{l}\text { ST } \\
(\%)\end{array}$} & \multirow{2}{*}{$\begin{array}{l}\text { GC } \\
(\%)\end{array}$} & \multirow{2}{*}{$\begin{array}{c}\mathrm{G} \\
(\%)\end{array}$} & \multirow{2}{*}{$\begin{array}{l}\text { SR } \\
(\%)\end{array}$} \\
\hline & $\begin{array}{c}\mathrm{U} \\
\left(\mathrm{kg} \mathrm{kg}^{-1}\right)\end{array}$ & $\begin{array}{c}\sigma_{\mathrm{p}} \\
(\mathrm{kPa})\end{array}$ & $\begin{array}{c}\mathrm{U} \\
\left(\mathrm{kg} \mathrm{kg}^{-1}\right)\end{array}$ & $\begin{array}{c}\sigma_{\mathrm{p}} \\
(\mathrm{kPa})\end{array}$ & $\begin{array}{c}\mathrm{U} \\
\left(\mathrm{kg} \mathrm{kg}^{-1}\right)\end{array}$ & $\begin{array}{c}\sigma_{\mathrm{p}} \\
(\mathrm{kPa})\end{array}$ & $\begin{array}{c}\mathrm{U} \\
\left(\mathrm{kg} \mathrm{kg}^{-1}\right)\end{array}$ & $\begin{array}{c}\sigma_{\mathrm{p}} \\
(\mathrm{kPa})\end{array}$ & & & & \\
\hline & \multicolumn{12}{|c|}{ Duas passadas do "forwarder" } \\
\hline a. Com compactação & - & - & - & - & - & - & $0,38^{(2)}$ & $237^{(2)}$ & 0 & 0 & 0 & 30 \\
\hline b. Com tendência a compactar & $0,36^{(3)}$ & $131^{(3)}$ & $0,38^{(4)}$ & $149^{(4)}$ & $0,36^{(5)}$ & $164^{(5)}$ & $0,33^{(6)}$ & $216^{(6)}$ & 50 & 80 & 90 & 70 \\
\hline \multirow[t]{2}{*}{ c. Sem compactação } & $0,35^{(3)}$ & $119^{(3)}$ & $0,32^{(7)}$ & $124^{(7)}$ & $0,30^{(8)}$ & $129^{(8)}$ & - & - & 50 & 20 & 10 & 0 \\
\hline & \multicolumn{12}{|c|}{ Oito passadas do "forwarder" } \\
\hline a. Com compactação & - & - & - & - & $0,41^{(8)}$ & $250^{(8)}$ & $0,39^{(3)}$ & $280^{(3)}$ & 0 & 0 & 10 & 50 \\
\hline b. Com tendência a compactar & $0,36^{(3)}$ & $131^{(3)}$ & $0,37^{(9)}$ & $154^{(9)}$ & $0,37^{(5)}$ & $186^{(5)}$ & $0,33^{(3)}$ & $216^{(3)}$ & 50 & 95 & 90 & 50 \\
\hline c. Sem compactação & $0,35^{(3)}$ & $119^{(3)}$ & $0,34^{(10)}$ & $116^{(10)}$ & - & - & - & - & 50 & 5 & 0 & 0 \\
\hline
\end{tabular}

${ }^{(1)}$ ST: sem tráfego do "forwarder"; GC: presença de galhada+casca; G: presença de galhada; SR: sem resíduo. (2)Média de 6 amostras. ${ }^{(3)}$ Média de 10 amostras. ${ }^{(4)}$ Média de 16 amostras. ${ }^{(5)}$ Média de 18 amostras. ${ }^{(6)}$ Média de 14 amostras. ${ }^{(7)}$ Média de 4 amostras. ${ }^{(8)}$ Média de 2 amostras. ${ }^{(9)}$ Média de 19 amostras. ${ }^{(10)}$ Uma amostra.

das amostras; e na condição de solo sem resíduo (SR), $50 \%$ das amostras foram compactadas, o que indica que, também nesta região, a condição galhada + casca (GC) foi a mais eficiente em minimizar o efeito do tráfego do "forwarder" sobre a estrutura do solo.

Tanto na região do Rio Doce quanto na de Guanhães, a condição solo sem resíduo (SR) foi a condição em que ocorreu maior compactação do solo, em razão do tráfego do "forwarder", enquanto as condições de galhada + casca (GC) e galhada (G), mesmo na condição de tráfego mais intensa (oito passadas), foram as condições mais eficientes na distribuição das pressões aplicadas ao solo pelo "forwarder", o que foi evidenciado pelo menor aumento das pressões de preconsolidação, menor compactação do solo, com conseqüente preserva- ção da sua estrutura, em razão da presença da camada de resíduo, que teve efeito minimizador da compactação do solo nestas regiões, fato que corrobora os resultados obtidos por McMahon \& Evanson (1994) e Seixas et al. (1998).

\section{Conclusões}

1. Os resíduos florestais minimizam a compactação do solo causada pelo tráfego do "forwarder".

2. O solo sem resíduo (SR) é mais suscetível à compactação.

3. A presença de galhada + casca (GC) é a condição que oferece maior resistência à compactação.

4. O aumento da intensidade do tráfego do "forwarder" causa maior compactação. 


\section{Referências}

ARVIDSSON, J.; SJÖBERG, E.; AKKER, J.J.H. van den. Subsoil compaction by heavy sugarbeet harvesters in Southern Sweden: III. Risk assessment using a soil water model. Soil and Tillage Research, v.73, p.77-87, 2003.

BLAKE, G.R.; HARTGE, K.H. Particle density. In: KLUTE, C. (Ed.). Methods of soil analysis: physical and mineralogical methods. $2^{\text {nd }}$ ed. Madison: American Society of Agronomy, 1986. v.1, p.377382. (Agronomy monograph, 9).

CANILLAS, E.C.; SALOKHE, V.M. A decision support system for compaction assessment in agricultural soils. Soil and Tillage Research, v.65, p.221-230, 2002.

CHAMEN, T.; ALAKUKKU, L.; PIRES, S.; SOMMER, C.; SPOOR, G.; TIJINK, F.; WEISSKOPF, P. Prevention strategies for field traffic-induced subsoil compaction: a review. Part 2. Equipment and field practices. Soil and Tillage Research, v.73, p.161-174, 2003.

CZYZ, E.A. Effects of traffic on soil aeration, bulk density and growth of spring barley. Soil and Tillage Research, v.79, p.153166, 2004.

DAY, P.R. Particle fractionation and particle size analysis. In: KLUTE, C.A. (Ed.). Methods of soil analysis: physical and mineralogical methods. $2^{\text {nd }}$ ed. Madison: American Society of Agronomy, 1986. v.1, p.545-567. (Agronomy monograph, 9).

DIAS JUNIOR, M. de S. Compression of three soils under longterm tillage and wheel traffic. 1994. 114p. Tese (Doutorado) Michigan State University, East Lansing.

DIAS JUNIOR, M. de S.; FERREIRA, M.M.; FONSECA, S.; SILVA, A.R.; FERREIRA, D.F. Avaliação quantitativa da sustentabilidade estrutural dos solos em sistemas florestais na região de Aracruz-ES. Revista Árvore, v.23, p.371-380, 1999.

DIAS JUNIOR, M. de S.; GOMES, A.N.; ANDRADE, S.C.; AZEVEDO, M.R. Avaliação da sustentabilidade da estrutura de Argissolos em sistemas florestais. Cerne, v.8, p.103-114, 2002.

DIAS JUNIOR, M. de S.; LEITE, F.P.; LASMAR JÚNIOR, E.; ARAÚJO JUNIOR, C.F. Traffic effects on the soil preconsolidation pressure due to eucalyptus harvest operations. Scientia Agricola, v.62, p.248-255, 2005.

DIAS JUNIOR, M. de S.; LEITE, F.P.; WINTER, M.E.; PIRES, J.V.G. Avaliação quantitativa da sustentabilidade estrutural de um Latossolo Amarelo cultivado com eucalipto na região de Peçanha MG. Revista Árvore, v.27, p.343-349, 2003.
DIAS JUNIOR, M. de S.; PIERCE, F.J. O processo de compactação do solo e sua modelagem: revisão de literatura. Revista Brasileira de Ciência do Solo, v.20, p.175-182, 1996.

DIAS JUNIOR, M. de S.; PIERCE, F.J. A simple procedure for estimating preconsolidation pressure from soil compression curves. Soil Technology, v.8, p.139-151, 1995.

HAMZA, M.A.; ANDERSON, W.K. Soil compaction in cropping systems: a review of the nature, causes and possible solutions. Soil and Tillage Research, v.82, p.121-145, 2005.

HOLTZ, R.D.; KOVACS, W.D. An introduction to geotechnical engineering. Englewood Cliffs: Prentice-Hall, 1981. 733p.

McMAHON, S.; EVANSON, T. The effect of slash cover in reducing soil compaction resulting from vehicle passage. Logging Industry Research Organisation Report, v.9, p.1-8, 1994.

PENG, X.H.; HORN, R.; ZHANG, B.; ZHAO, Q.G. Mechanisms of soil vulnerability to compaction of homogenized and recompacted Ultisols. Soil and Tillage Research, v.76, p.125-137, 2004.

RAIJ, B. van; QUAGGIO, J.A. Métodos de análise de solo para fins de fertilidade. Campinas: Instituto Agronômico, 1983. 16p. (Circular, 63).

RAPER, R.L. Agricultural traffic impacts on soil. Journal of Terramechanics, v.42, p.259-280, 2005.

SEIXAS, F.; KOURY, C.G.G.; RODRIGUES, F.A. Determinação da área impactada pelo tráfego de forwarder com uso de GPS. Scientia Forestalis, v.68, p.178-187, 2003.

SEIXAS, F.; OLIVEIRA JÚNIOR, E.D.; SOUZA, C.R. Efeito da camada de resíduos florestais na compactação do solo causada pelo transporte primário da madeira. Scientia Forestalis, v.54, p.9-16, 1998.

SERVADIO, P.; MARSILI, A.; VIGNOZZI, N.; PELLEGRINI, S.; PAGLIAI, M. Effects on some soil qualities in central Italy following the passage of four wheel drive tractor fitted with single and dual tires. Soil and Tillage Research, v.84, p.87-100, 2005.

SILVA, A.R. Modelagem da capacidade de suporte de carga e quantificação dos impactos causados pelas operações mecanizadas na estrutura de um Latossolo Amarelo cultivado com cafeeiros. 2003. 39p. Dissertação (Mestrado) - Universidade Federal de Lavras, Lavras.

TAYLOR, D.W. Fundamentals of soil mechanics. New York: Wiley, 1948. 700p.

WOOD, R.K.; REEDER, R.C.; MORGAN, M.T.; HOLMES, R.G. Soil physics properties as affected by grain cart traffic. Transactions of the American Society of Agricultural Engineers, v.36, p.11-14, 1993. 